Z Rheumatol 2010 69 :657-657 DOI 10.1007/s00393-010-0645-x Online publiziert: 22. Juli 2010

(c) Springer-Verlag 2010

\author{
S. George \\ Deutscher Verband der Ergotherapeuten e.V., Karlsbad
}

\title{
Defizite der ergotherapeutischen Routineversorgung bei rheumatischen Erkrankungen
}

\section{Zum Beitrag}

Thieme H, Löffler K, Borgetto B (2009) Defizite der ergotherapeutischen Routineversorgung bei rheumatischen Erkrankungen. Z Rheumatol 2010 · 69:435-442

Sehr geehrter Herr Thieme, sehr geehrte Frau Löffler, sehr geehrter Herr Professor Borgetto,

vielen Dank für Ihren Artikel „Defizite der ergotherapeutischen Routineversorgung bei rheumatischen Erkrankungen“, der aus unserer Sicht einen wichtigen und gelungenen Beitrag zur Versorgungsforschung im Bereich der nichtmedikamentösen Therapieverfahren darstellt.

Wir haben lediglich eine Anmerkung: Der Satz „Eine grundsätzliche Erweiterung der Heilmittelrichtlinien sollte eine Unterversorgung von rheumatisch erkrankten Patienten vermeiden“, im Fazit auf Seite 7, könnte von Leserinnen und Lesern, die mit den Heilmittelrichtlinien (HMR) nicht vertraut sind, so interpretiert werden, als würden die HMR einer flächendeckenden Versorgung von Patienten mit rheumatischen Erkrankungen entgegen stehen.

Wir möchten daher ausdrücklich darauf hinweisen, dass dies nicht der Fall ist: Nach der gültigen Fassung der HMR (vom 16.03.2004) kann Ergotherapie für diese Patienten ganz regulär verordnet werden, und zwar je nach Indikation unter den Diagnosegruppen SB 1 (Wirbelsäulenerkrankungen), SB 4 (Gelenkerkrankungen), SB 5 (Gelenkerkrankungen/ Störungen der Gelenkfunktion) und zum Teil außerdem unter SB 7 [Erkrankungen mit Gefäß-, Muskel- und Bindegewebs- beteiligung, insbesondere systemische Erkrankungen (betrifft z. B. Lupus erythematodes)]. Für jede dieser Diagnosegruppen stehen unterschiedliche Verordnungsmengen zur Verfügung, die von sechs (SB 4 bei kurzzeitigem Behandlungsbedarf) bis hin zu 30 Behandlungseinheiten (etwa bei den Indikationen der Diagnosegruppe $\mathrm{SB} 7$ ) reichen.

Sollten die dort vorgesehenen Verordnungsmengen des Regelfalls nicht ausreichen, bieten die HMR zudem explizit die Möglichkeit zur Verordnung außerhalb des Regelfalls.

In diesem Sinne hoffen wir, dass Ihr Artikel dazu beiträgt, dass die vorhandenen Möglichkeiten zur Versorgung von rheumatisch erkrankten Patienten mit Ergotherapie künftig besser genutzt werden.

Mit freundlichen Grüßen

Sabine George

\section{Korrespondenzadresse}

Sabine George

Deutscher Verband der Ergotherapeuten e.V.

Postfach 2208

76303 Karlsbad

s.george@dve.info 